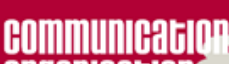
Oryanllatín.

\section{Communication et organisation}

Revue scientifique francophone en Communication organisationnelle

59 | 2021

La communication constitutive des organisations : émergence et innovations

\title{
PIJOAN Natacha et PLANE Jean-Michel (dir.), 2020. Approches critiques des organisations
}

Caen : Éditions Management \& Société

Eloria Vigouroux-Zugasti

\section{OpenEdition}

\section{Journals}

Édition électronique

URL : https://journals.openedition.org/communicationorganisation/9588

DOI : 10.4000/communicationorganisation.9588

ISSN : $1775-3546$

Éditeur

Presses universitaires de Bordeaux

Édition imprimée

Date de publication : 1 juin 2021

Pagination : 289-291

ISSN : $1168-5549$

Référence électronique

Eloria Vigouroux-Zugasti, «PIJOAN Natacha et PLANE Jean-Michel (dir.), 2020. Approches critiques des organisations », Communication et organisation [En ligne], 59 | 2021, mis en ligne le 01 juin 2021,

consulté le 10 décembre 2022. URL : http://journals.openedition.org/communicationorganisation/ 9588 ; DOI : https://doi.org/10.4000/communicationorganisation.9588 


\title{
Recensions
}

\author{
PIJOAN, Natacha et PLANE, Jean-Michel (dir.), 2020. Approches \\ critiques des organisations.
}

Caen : Management \& Société

\section{Par Eloria Vigouroux-Zugasti}

Approches critiques des organisations est proposé par Natacha Pijoan et Jean-Michel Plane, respectivement maîtresse de conférences HDR à l'université Paul-Valéry Montpellier 3 et Professeur des universités dans ce même établissement. L'ouvrage propose des regards croisés sur les approches critiques des organisations, afin de rendre hommage au Professeur Alain Briole. A un moment de leur carrière, les contributeurs ont rencontré, discuté, voire travaillé avec le Professeur. Les travaux réunis dans cet ouvrage représentent un " mélange » rappelant la singularité de la carrière d'Alain Briole et la grande variété de ses thématiques de recherche, qu'elles concernent le milieu organisationnel (employabilité, évolution des métiers, relation à l'emploi, etc.) ou des sujets plus larges, liés au développement humain, à la considération sociale de certaines populations (vulnérabilité, seniors et femmes au travail) ou encore à l'innovation sociale. Une carrière prolifique qui est aujourd'hui mise à l'honneur, non seulement pour sa grande liberté scientifique, mais également pour son importance dans la recherche collective en SHS.

L'approche critique est fortement revendiquée, dès la préface de l'ouvrage (Natacha Pijoan \& Jean-Michel Plane) et se retrouve à travers l'ensemble des contributions. Dans le chapitre qui leur est consacré, Camille Ricaud, Jean-Pierre Neveu et Jacques Jaussaud parlent de l'importance "d'oxygéner " la recherche en sciences humaines. Pour les auteurs, cela passe par une démarche parfois qualifiée de " punk » (p. 64), c'est-à-dire une volonté affirmée de remise en cause de l'ordre scientifique, plus particulièrement des codes et des présupposés de chaque discipline pour en faire évoluer les positions, les paradigmes et les thèmes de recherche. Cette démarche critique se traduit de plusieurs manières au sein de l'ouvrage. Nous retrouvons ainsi l'analyse de thématiques sensibles et peu documentées (l'autiste au prisme des outils de gestion, les Objectifs de Développement Durable (ODD) au sein d'entreprises du CAC 40, etc.), ou de thématiques émergentes habituellement traitées de manière " positive » (les limites de l'organisation libérée, les écueils de la gestion de la diversité en entreprise, etc.).

L'ouvrage se compose de quatre parties. La première, contenant sept chapitres, est consacrée aux approches interdisciplinaires et critiques des organisations. Nous 
retrouvons ainsi des questions relatives à l'épistémologie de la transdisciplinarité (Odile Uzan), à la sociologie d'intervention (Manuel Boucher \& Yves Gilbert) ou, entre autres, au leadership (Adda Benslimane \& Jean-Michel Plane ; Adnane Chader \& Hayat Sbai). La deuxième partie comporte six chapitres dédiés à l'appréhension des organisations et de leurs acteurs dans un contexte en mutation. Les contributions abordent notamment la question de la RSE (Jacques Igalens ; Hajar El Bahri), du management (Michelle Duport et al.) ou encore de l'âge en entreprise (Ketty Bravo et al.). Les sept chapitres de la troisième partie traitent des différents aspects de l'entreprise : le soutien organisationnel en contexte procédural (Serge Ndayirata \& Sophia Belghiti-Mahut), le servant leadership (Florence Noguera \& Laurent Cappelletti), la mutation des universités et ses conséquences en termes managériaux (Laurence Dreyfuss \& Sylvie Rascol-Boutard), etc. S’ensuit une postface dédiée à l'ensemble du travail scientifique du Professeur Alain Briole, qui conclut cet ouvrage d'hommage à sa carrière.

Approches critiques des organisations est un ouvrage particulièrement touchant et saisissant. Touchant, car la démarche collective de reconnaissance de l'œuvre d'un collègue s'avère poignante dès les premières lignes de la préface. L’ouvrage se révèle saisissant, d'autre part, car à travers les approches critiques des thématiques et la variété des sujets abordés, il met en perspective la richesse que représente l'étude du milieu organisationnel en sciences humaines et sociales, richesse d'ailleurs mise en abyme dans la présentation du parcours d'Alain Briole.

Le choix d'une perspective critique représente l'un des éléments les plus originaux de cet ouvrage. En effet, tout en évoquant des sujets qui ont parfois été documentés dans de nombreuses publications, à l'instar du sensemaking en milieu hospitalier (Ariel Eggrickx, p. 267), l'approche par la dimension critique permet de renouveler les paradigmes et les constructions scientifiques dont ces thématiques ont déjà fait l'objet. De plus, l'ouvrage aborde des sujets émergeants : l'organisation libérée (Zino Khelfaoui, p. 99), le leadership spirituel (Mihaela-Alexandra Tudor \& Stefan Bratosin, p. 175), etc. Ces thèmes ont été abordés dans la littérature dite " grise " et tendent à être présentés comme de "nouveaux modèles organisationnels ». Ils suscitent un certain engouement dans le milieu du management et de l'entreprenariat. Or, leur caractère émergeant permet-il d'avoir assez de recul pour saisir l'ensemble de leurs enjeux et surtout, de leurs limites ? Les analyses critiques présentées dans le présent ouvrage proposent, très justement, une prise de recul pertinente et une réflexivité scientifique, qui viennent en contrepoint des discours médiatiques promouvant ces nouveaux modèles managériaux.

Approches critiques des organisations s'impose comme un ouvrage particulièrement riche. Son originalité, sa justesse et la qualité rédactionnelle des contributions en font un ouvrage indispensable pour les chercheurs se consacrant à l'étude du milieu organisationnel, qu'elle soit inscrite en sciences de gestion, en sciences de 
l'information et de la communication ou, plus globalement, en sciences humaines et sociales. Il présente également un atout certain aussi bien pour les étudiants souhaitant approfondir leurs connaissances que pour les enseignants aspirant à diversifier leurs contenus pédagogiques.

Référence : PIJOAN Natacha et PLANE Jean-Michel (dir.), 2020. Approches critiques des organisations. Caen : Éditions Management \& Société. ISBN 978-2-37687-373$0,34 €$.

Eloria Vigouroux-Zugasti est docteure en sciences de l'information et de la communication, A.T.E.R. à l'Université Gustave Eiffel et chercheure associée au laboratoire MICA (EA 4426) et au laboratoire DICEN-IDF (EA 7339). 